Boise State University

ScholarWorks

$5-1-2009$

\title{
Observation and Modeling of Source Effects in Coda Wave Interferometry at Pavlof Volcano
}

Matthew M. Haney

Boise State University

Kasper van Wijk

Boise State University

Leiph A. Preston

Sandia National Laboratories

David F. Aldridge

Sandia National Laboratories 


\title{
Observation and modeling of source effects in coda wave interferometry at Pavlof volcano
}

\author{
Matthew M. Haney, U.S. Geological Survey Alaska Volcano Observatory \\ KASPER VAN WIJK, Boise State University \\ Leiph A. Preston and David F. Aldridge, Sandia National Laboratories
}

$S^{\circ}$

orting out source and path effects for seismic waves at volcanoes is critical for the proper interpretation of underlying volcanic processes. Source or path effects imply that seismic waves interact strongly with the volcanic subsurface, either through partial resonance in a conduit (Garces et al., 2000; Sturton and Neuberg, 2006) or by random scattering in the heterogeneous volcanic edifice (Wegler and Luhr, 2001). As a result, both source and path effects can cause seismic waves to repeatedly sample parts of the volcano, leading to enhanced sensitivity to small changes in material properties at those locations. The challenge for volcano seismologists is to detect and reliably interpret these subtle changes for the purpose of monitoring eruptions.

We examine seismic records of repeating explosions from Pavlof volcano, Alaska, during its 2007 eruption. Repetitive explosions are typical of Strombolian-style eruptions and allow measurement of relative time shifts between similar latearriving phases using the technique called coda wave interferometry (Snieder et al., 2002). The measurements enable the detection of small changes in the volcanic interior of Pavlof. We are able to resolve an increase in the relative traveltime change of late-arriving seismic waves on the order of $0.3 \%$ over the course of two weeks. Based on the spectra of the explosions, their location inside the magma conduit, previous studies of Pavlof volcano, and 3D seismic modeling, we argue the most likely scenario is one in which the velocity and/or the geometry of the conduit changes. This demonstrates the sensitivity of coda wave interferometry to source effects, in addition to path effects, at volcanoes.

Small changes were observed during the 2007 eruption of Pavlof volcano, located along the continental portion of the Aleutian arc (Figure 1). A series of long-period (LP) earthquakes and tremor bursts on 14 August 2007 marked the reawakening of Pavlof from an 11-year period of repose. The 2007 eruption of Pavlof persisted for roughly one month, until September 13, and was characterized by explosions, tremors, lahars, and lava flows on the eastern slope (Figure 2). Brittle failure earthquakes did not occur during the eruption, providing no data for conventional methods like earthquake location or seismic tomography. Activity at Pavlof was monitored by the USGS Alaska Volcano Observatory, which operates a local seismic network of five short-period seismometers around the volcano (Figure 1).

Coda wave interferometry (CWI) makes it possible to track small time-lapse variations in repeating explosions observed during the Pavlof eruption. CWI has its roots in pioneering work on earthquake multiplets (Poupinet et al., 1984) and the doublet method (Roberts et al., 1992) and is sensitive to several types of change in the subsurface (Snieder,

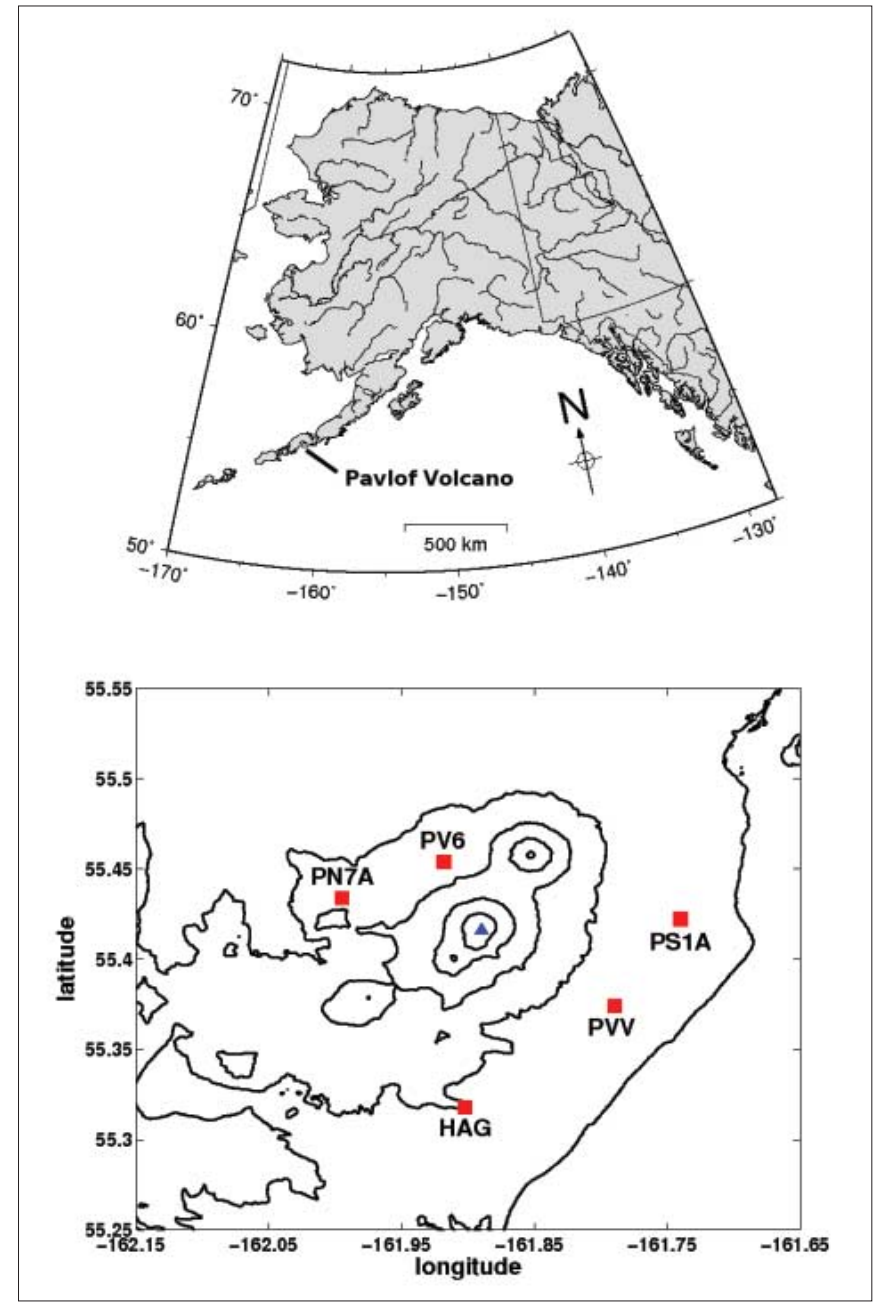

Figure 1. (top) Pavlof volcano, on the western end of the Alaska Peninsula. (bottom) The Pavlof volcano seismic network, consisting of four vertical-component, short-period L4 seismometers and one 3-C, short-period seismometer (PVG). The summit of Pavlof is shown as a blue triangle.

2006). A common change is a uniform reduction in seismic velocity between scatterers distributed throughout a volcano. This change causes a progressive time lag in time-lapse seismic signals. Previous studies of CWI at volcanoes by Wegler et al. (2006) and Pandolfi et al. (2006) have interpreted observations within this model. The time-lapse signal is thus explained by a changing path effect brought about by random scattering within a volcano. In contrast, we find that similar observations during the 2007 eruption of Pavlof can alternatively be explained by a changing source effect. Thus, care must be taken in the interpretation of CWI results since different conclusions can be made depending on whether source 


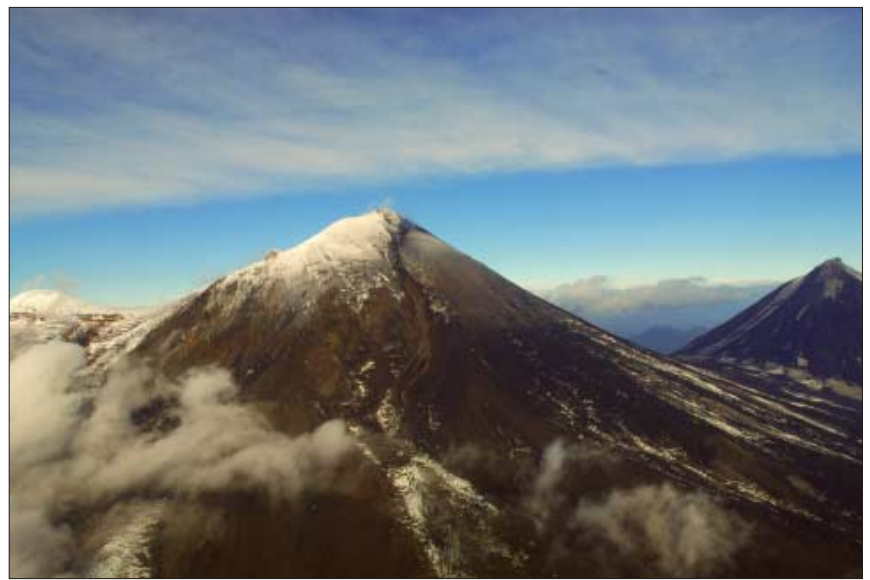

Figure 2. Pavlof volcano viewed from the east after the eruption on 19 September 2007, with the path taken by lava flows in the foreground. Photograph by Chris Waythomas, USGS Alaska Volcano Observatory.

or path effects dominate the wavefield. When source effects are dominant, as is likely the case at Pavlof, CWI may be used to precisely monitor the evolution of the volcanic conduit during the eruption.

\section{Repeating explosions}

The eruption at Pavlof produced many (300-400) repeating explosions per day. To find these repeating explosions, we first selected a master explosion that occurred on 29 August 2007 at approximately 16:41:35 UTC. The master explosion itself is not particularly special, other than standing out clearly from the background noise and being separated in time from other events. We identify repeating explosions from seismic recordings by cross-correlating the early portion (first-arriving $8 \mathrm{~s}$ ) of the master explosion with 8-s moving windows of the continuous data. For each time sample, we compute the zero-lag correlation coefficient and save the time samples when the coefficient exceeds 0.85 . We identify these time samples as the repeating explosions. This technique is related to the method described by Petersen et al. (2006), except that Petersen et al. use spectral-coherence with a 0.9 threshold instead of a temporal-coherence correlation coefficient to measure similarity.

As demonstrated by McNutt (1986), the repeating explosions once identified may be stacked to improve the signalto-noise ratio. This is important since the application of CWI requires on-scale recording of late-arriving seismic waves above the background level. Shown on the left in Figure 3 are the radial, vertical, and transverse stacks of the repeating explosions occurring between 29 August 2007 at 18:00:00 UTC and 30 August 2007 at 06:00:00 UTC for station PV6, the only three-component station in the Pavlof network. The radial and transverse stacks are obtained by rotating the horizontal components toward the summit of Pavlof. Throughout this paper, we consider a sequence of 25 nonoverlapping 12-hour time windows and identify each time window by its center time. The time of the stacks shown in Figure 3 is thus taken as 30 August 2007 at 00:00:00 UTC. Also plotted in Figure 3 are the spectra of the vertical stacks at stations PV6

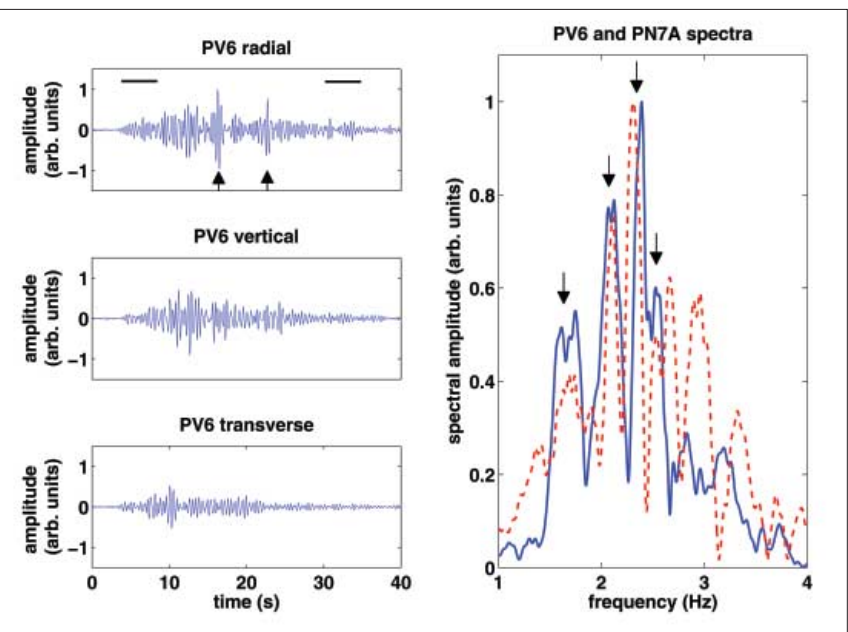

Figure 3. (left) Radial, vertical, and transverse stacks of particle velocity from repeating explosions at station PVG during the 12-hour time window centered around 30 August 2007 at 00:00 UTC. Upward-pointing arrows highlight large amounts of late-arriving radial energy at PVG. The (black) horizontal lines show two time windows discussed further in Figure 4. (right) Comparison of the amplitude spectra for the vertical stacks at stations PVG (solid blue) and PN7A (dashed red). Common peaks are shown by downwardpointing arrows.

and PN7A. Note that, prior to stacking, we low-pass filter the individual explosions below $4 \mathrm{~Hz}$ to accentuate the dominant frequency band of these events. The waveforms and spectra in Figure 3 suggest a dominance of source effects over path effects for the LP explosions at Pavlof. This inference is based on: (1) the two high-amplitude, late-arriving wave packets in the PV6 radial stack (marked by upward arrows in Figure 3), which show coherent energy streaming radially outward from the summit at late times; and, most importantly, (2) the common spectral peaks in the vertical stacks at the two stations PV6 and PN7A (shown by downward arrows). The existence of common spectral peaks between stations is a well-known test for establishing the dominance of source effects at volcanoes (Chouet, 1996). The common spectral peaks between 1.5 and $2.5 \mathrm{~Hz}$ are indicative of resonance in the magma conduit at Pavlof induced by the explosions (Garces et al.).

\section{Time-lapse time shifts}

We perform CWI on the 25 sequential waveform stacks at stations PV6 and PN7A (Figure 1). At PV6, we use radial stacks, while at PN7A we use vertical stacks since PN7A is a single-component seismometer. These 25 sequential stacks begin on 30 August 2007 at 00:00:00 UTC (center time) and end on 11 September 2007 at 00:00:00 UTC. By that time, the eruption at Pavlof waned, and large numbers of repeating explosions were no longer recorded.

The top panel of Figure 4 compares the early arrivals for the two stacks of the radial component at PV6 taken on 30 August 2007 at 00:00:00 UTC and 3 September 2007 at 12:00:00 UTC. The two traces match each other well, which is to be expected since the similarity of the early waveforms (first-arriving $8 \mathrm{~s}$ ) is the criterion for selecting the repeating explosions, as described previously. The middle panel of Fig- 

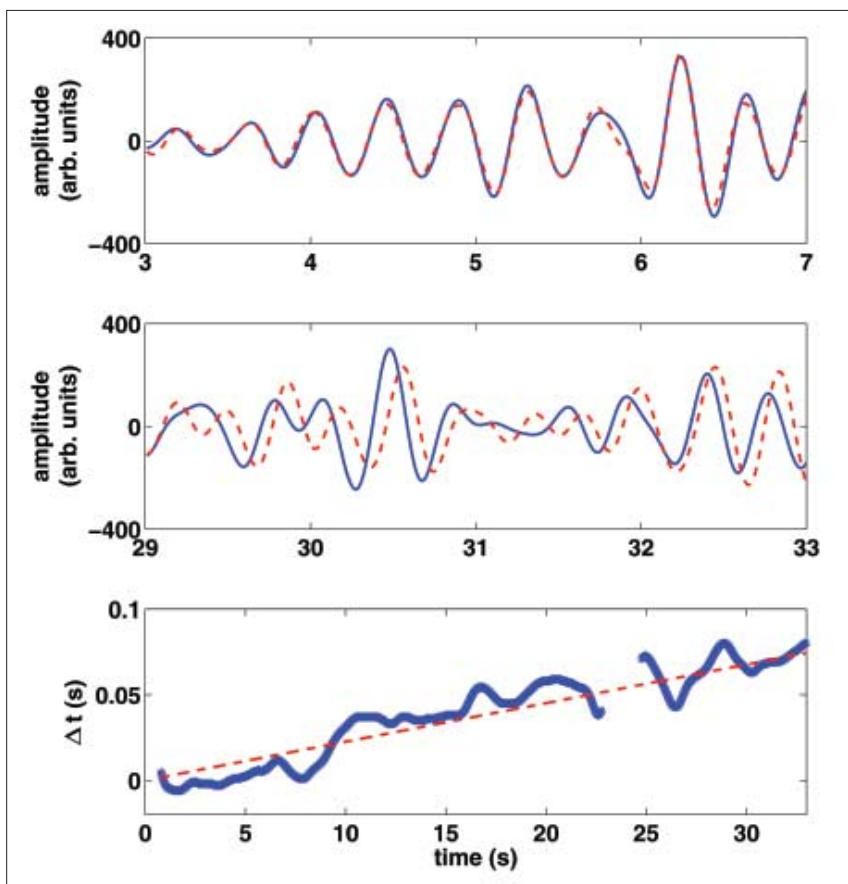

Figure 4. (top) Comparison of a stack of particle velocity from repeating explosions at Pavlof (blue solid line) with a stack from 4.5 days later (red dashed line) at early times. (middle) Same as the top panel for late times. (bottom) The relative time shifts as a function of time, and the linear fit to the data (red dashed line). As in Wegler et al., values of $\Delta \mathrm{t}$ are not used if the correlation coefficient is less than 0.7 ; this is the reason for the small gap at around $23 \mathrm{~s}$.

ure 4 shows the same comparison for the late-arriving waves. In contrast to the early arriving waves, the late-arriving waves do not match in time for the two stacks. The waveforms from the later stack are to a great degree a time-shifted (timelagged) version of the waveforms from the earlier stack.

The time lag between two traces can be quantified by taking time-windowed crosscorrelations of the traces and finding the lag time of the maximum correlation coefficient as a function of time (Snieder et al.). We perform this process on stacks from both PV6 and PN7A while always using the first stack from the respective station (August 30 at 00:00:00 UTC) as one of the traces. In this way, the first stack is a baseline for measuring all future changes. The process is illustrated in the bottom panel of Figure 4 for station PV6. Simple physical models (Snieder et al.; Pandolfi et al.; Wegler et al.) predict that the measured time lag between the two traces, $\Delta t$, should be a linear function of the traveltime $t$

$$
\Delta t=m t
$$

with a slope $m$ that depends on the type and degree of physical change happening between the times of the two measurements. We discuss later the interpretation of $m$ and give examples of the associated physical models. The bottom panel of Figure 4 demonstrates that the time shift (time delay) grows with increasing traveltime for the two traces. We fit the observed time shifts according to the linear relation in Equation 1. Certainly, deviations from the linear assumption exist in the observed time shifts; we attempt, however, to make

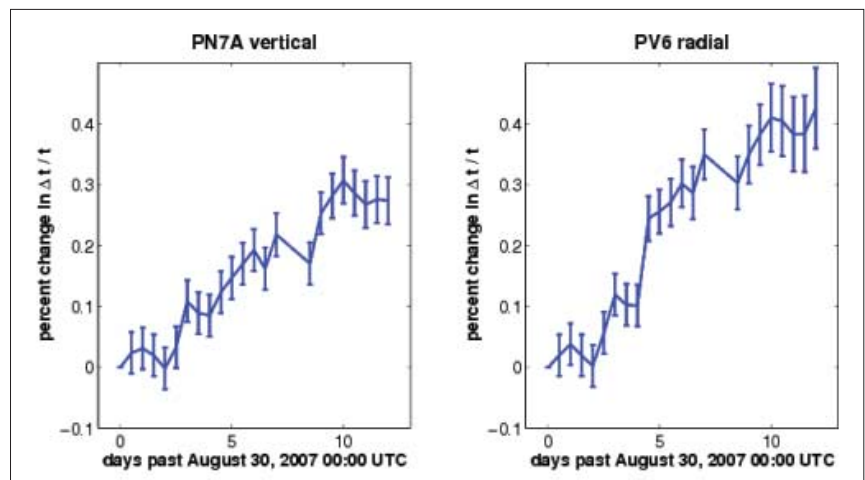

\section{closing conduit lengthening conduit}

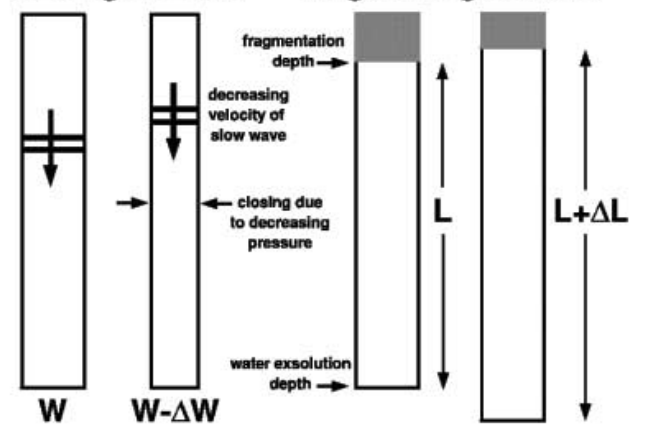

Figure 5. (top) Measured relative traveltime changes for stations PN7A and PVG over the final two weeks of the eruption, along with their associated errors. Note that the time period between 6 and 7 September is omitted since few repeating explosions $(<50)$ occurred during that time. (bottom) Two interpretations for the observed increase in relative traveltime (see text for details).

as simple an interpretation as possible and so use the linear relation.

We plot the measured slopes $m=\Delta t / t$, called the relative traveltime change, in the top panels of Figure 5 for stations PN7A and PV6 along with error bars on the estimates of $\Delta t / t$. From these plots, we find that the relative traveltime change progressively increased over the last two weeks of the eruption at Pavlof. Although the magnitude of the change measured at PV6 is slightly higher, the relative traveltime change at both stations had increased and reached a value of roughly $0.3 \%$ by 11 September 2007.

\section{Interpretation}

The measured relative traveltime changes are usually interpreted using the relation

$$
\Delta t / t=-\Delta v / v
$$

where the slope $m$ mentioned previously is taken to be $m=-\Delta v / v$ and $v$ is the seismic velocity. This relation assumes a uniform velocity change everywhere in the subsurface. From this relation, the $0.3 \%$ traveltime change measured at Pavlof could be interpreted as a $-0.3 \%$ average reduction in seismic velocity within the entire edifice of the volcano. The change is assumed to take place over the entire volcano since multiple scattering from distributed random heterogeneities in the volcanic interior is assumed to give rise to the late- 
arriving waves.

However, the inferred dominance of source effects (Figure 3) argues against scattering from distributed random heterogeneities as the main cause for the late-arriving waves at Pavlof. This may be the result of the relatively low frequencies of the repeating explosions $(-2.5 \mathrm{~Hz})$. In other applications of CWI at volcanoes, Wegler et al. and Pandolfi et al. bandpass filtered their signals in frequency bands of 4-12 Hz and 4-15 Hz, respectively. In contrast, we have low-pass filtered the repeating explosions below $4 \mathrm{~Hz}$. Scattering from smallscale random heterogeneities (Rayleigh scattering) is known to become weaker at lower frequencies. Additionally, the explosions at Pavlof are located within the magma conduit, a region that has a strong material contrast with the rest of the volcano. Neither Wegler et al. nor Pandolfi et al. employed seismic sources located within conduits: Wegler et al. used active seismic sources at the surface of Merapi, and Pandolfi et al. used repeating volcano-tectonic earthquakes beneath Vesuvius. Further supporting the dominance of source effects, the waveforms of explosions from previous eruptions of Pavlof have been interpreted in terms of resonance in the uppermost section of the conduit.

We thus interpret the late-arriving waves at PV6 and PN7A to be primarily due to waves that are partially trapped in the magma conduit until they couple into the encasing solid and propagate to the seismic stations. As shown by Landro and Stammeijer (2004), the perturbation in traveltime of waves escaping from a layer can be related to perturbations in the thickness $z$ and velocity $v$ of the layer as

$$
\Delta t / t=\Delta z / z-\Delta v / v
$$

Similarly, when energy propagates primarily along the length of a volcanic conduit, the observed relative traveltime changes can be related to changes in the length $L$ and changes in the velocity of the conduit as

$$
\Delta t / t=\Delta L / L-\Delta v / v
$$

Therefore, the $0.3 \%$ relative traveltime change we measure at Pavlof could be either a $0.3 \%$ relative change in the length of the conduit, a $-0.3 \%$ relative change in the acoustic wave speed of the magma in the conduit, or a suitable combination of both types of change. In the following, we test one of these scenarios with numerical simulations using a 3D seismic model of volcanic explosions at Pavlof volcano.

\section{FD elastic seismic modeling}

For numerical modeling of seismic data at Pavlof, we employ a 3D staggered-grid, finite-difference (FD) implementation of the isotropic elastic velocity-stress system of equations (Graves, 1996; Preston et al., 2008). The velocity-stress formulation of the elastodynamic problem consists of a set of nine coupled, first-order partial differential equations for the three particle velocity vector components and the six independent stress tensor components. Sturton and Neuberg (2006) have previously applied 2D FD modeling in their study of conduit resonance at Soufriere Hills Volcano, Montserrat. We perform 3D FD modeling at Pavlof with topography data taken from a digital elevation model (DEM) with 2 arcsecond $(60 \mathrm{~m})$ lateral resolution. The topography data are freely available online at http://seamless.usgs.gov/. After simulating volcanic explosions from two conduit models, one with a slightly slower acoustic wave speed (-1\%), we perform CWI to estimate the change from time-lapse seismograms.

It is particularly important to include high-contrast interfaces, such as the rugged air-Earth and conduit-rock interface, when modeling seismic wavefields at volcanoes. FD modeling with a standard staggered grid in the presence of such interfaces is complicated by the fact that numerical instabilities often occur at locations of high material property contrasts (Haney, 2007). However, as pointed by Bohlen and Saenger (2006), second-order accurate modeling in time and space, called $\mathrm{O}(2,2)$, can be stabilized with the proper selection of material property averaging rules for density and shear modulus. For modeling seismic wavefields at Pavlof, we have adopted these rules and proceeded with $\mathrm{O}(2,2)$ standard staggered-grid numerical simulations. A better method is to employ order switching from $\mathrm{O}(2,4)$ within the Earth model to $\mathrm{O}(2,2)$ locally at high-contrast interfaces, thus optimizing overall stability and accuracy of the FD simulations (Preston et al.).

The 3D numerical model of Pavlof consists of three homogeneous subdomains: air $\left(V_{p}=350 \mathrm{~m} / \mathrm{s}, V_{s}=0 \mathrm{~m} / \mathrm{s}\right.$, and $\left.\rho=1 \mathrm{~kg} / \mathrm{m}^{3}\right)$; the shallow volcanic conduit beneath the summit of Pavlof $\left(V_{P}=500 \mathrm{~m} / \mathrm{s}, V_{S}=0 \mathrm{~m} / \mathrm{s}\right.$, and $\left.\rho=1750 \mathrm{~kg} / \mathrm{m}^{3}\right)$; and the subsurface of Pavlof $\left(V_{P}=3050 \mathrm{~m} / \mathrm{s}, V_{S}=1713.5 \mathrm{~m} / \mathrm{s}\right.$, and $\left.\rho=2300 \mathrm{~kg} / \mathrm{m}^{3}\right)$. The conduit properties are taken from Garces et al. for gas-saturated magma, and the subsurface properties are from McNutt. We set the shear-wave velocity of the magma in the conduit to zero as in Sturton and Neuberg. Although magma generally has a significant shear viscosity, this property can be ignored at low frequencies. The subsurface could be further defined with $3 \mathrm{D}$ variability if a tomographic model of Pavlof existed; however, for modeling of explosions within the conduit this is not of primary importance. The entire model is $401 \times 401 \times 201$ grid points in the $x, y, z$ directions with a uniform grid spacing of $30 \mathrm{~m}$. The conduit and subsurface together comprise the Earth model and are separated from the air by the rugged topographic surface. The conduit sits at the summit region of Pavlof and is defined by a parallelpiped with dimensions of 120,120 , and $300 \mathrm{~m}$ in the $x, y, z$ directions. The conduit is open to the atmosphere. In the numerical simulations, we set off a $1.5-\mathrm{Hz}$ explosion with a Ricker wavelet source-time function within the conduit. A time step of $4 \mathrm{~ms}$ was used, which is approximately $70 \%$ of the Courant-Friedrichs-Levy (CFL) stability condition for a homogeneous wholespace (Aldridge and Haney, 2008). The simulation executes 8000 time steps, thereby modeling $32 \mathrm{~s}$ of wave propagation.

\section{Numerical simulation results}

Figures 6 and 7 show time slices of the 3D wavefield simula- 


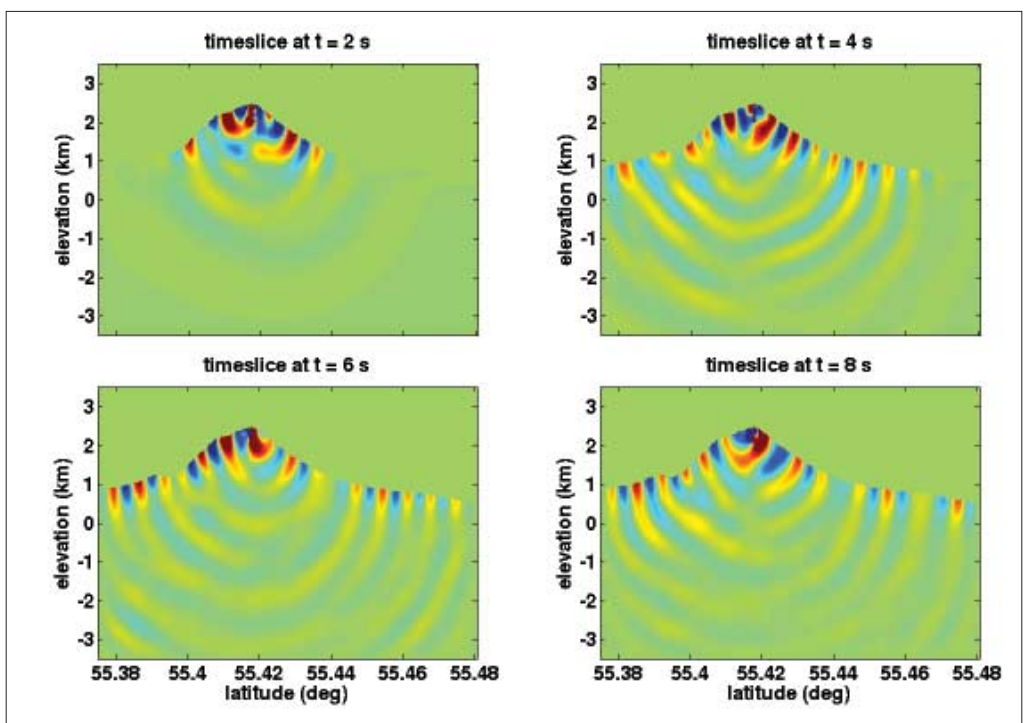

Figure 6. Vertical time slices of vertical particle velocity from a wavefield simulation of an explosion in the shallow conduit at Pavlof. The time slice is taken through the summit. The scattering from the high contrast at the conduit-rock interface causes a protracted wave train to emanate outward from the summit. The concentration of energy at the air-Earth interface points to a wavefield comprised mainly of surface waves. Although airwaves in the atmosphere are simultaneously modeled, their amplitudes have been muted to facilitate visualization of the wavefield within the volcano.

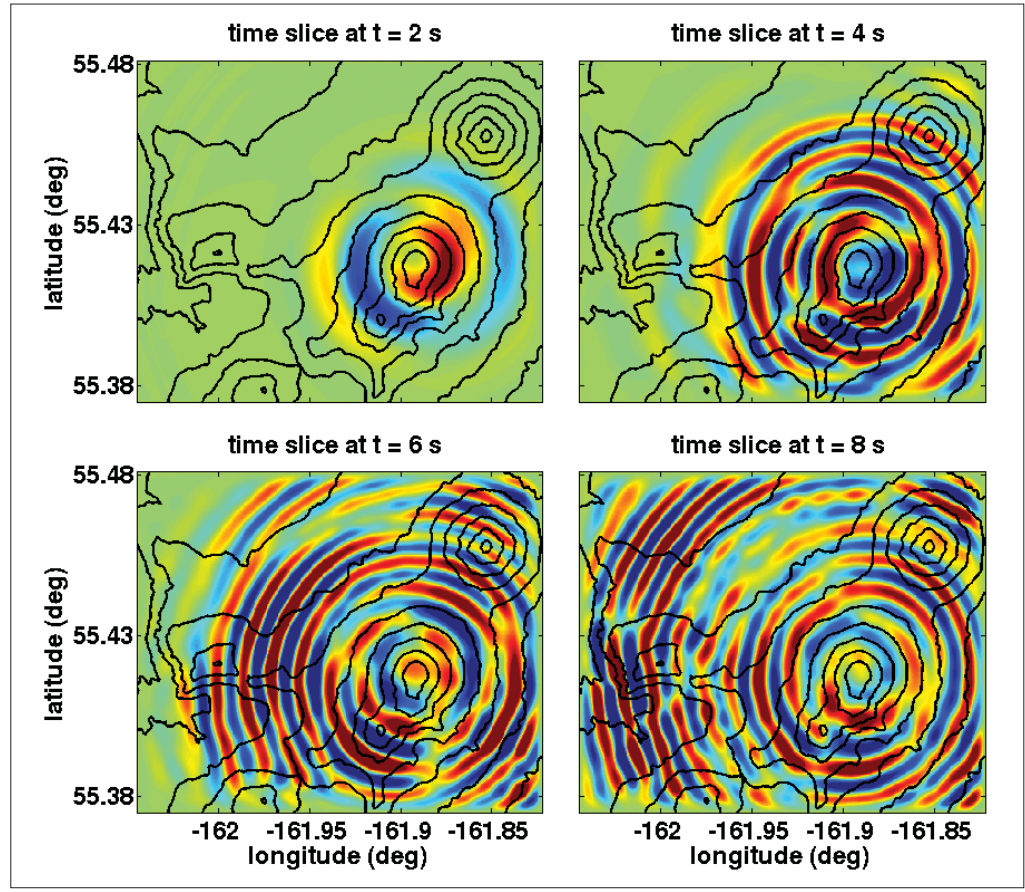

Figure 7. Horizontal time slices of vertical particle velocity from a wavefield simulation of an explosion in the shallow conduit at Pavlof. The time slice is taken at a depth of $200 \mathrm{~m}$ below sea level. Elevation contours are shown at intervals of $200 \mathrm{~m}$. Topographic focusing of the outward-propagating surface waves is evident, particularly on the westward-trending ridge from the summit.

tion at Pavlof. The wavefield is predominantly surface waves propagating outward from the summit region. The fine grid spacing within the subsurface ( -30 grid points per dominant shear wavelength) avoids the problem of unphysical diffractions from the topographic surface. Note that in Figure 6 we have muted the airwaves propagating in the atmosphere to focus attention on the seismic waves within the volcano. Although the shallow explosion source is impulsive, its location within the conduit causes the radiated wavefield to take on a "ringing" character due to repeated scattering at the high-contrast, conduit-rock interface. This source effect gives rise to late-arriving waves on the simulated radial component of station PV6 (Figure 8). Aldridge et al. (2008) successfully model similar seismic effects generated by a resonating air-filled underground tunnel, using the same 3D FD algorithm for isotropic elastic media.

The simulations shown in Figures 6 and 7 were repeated for the same numerical model, except the acoustic wave speed in the conduit was set $1 \%$ lower than the initial model $(495 \mathrm{~m} / \mathrm{s})$. From the simulated time-lapse radial seismograms at station PV6 (Figure 8), CWI can provide an estimate of the velocity change within the conduit. The relative velocity change from CWI, $-0.6 \%$, is smaller than the true change, $-1.0 \%$. The fact that CWI underestimates the true change in such a situation has been discussed previously by Khatiwada et al. (2008) and is related to the propagation path taken by the waves outside of the conduit, where no difference exists between the two numerical models. Thus, the $0.3 \%$ relative traveltime change observed at Pavlof over the final two weeks of the eruption (Figure 5) can be interpreted instead as a larger change in the acoustic-wave speed of the conduit.

\section{Discussion}

Equation 4 leads to three possible origins of the time-lapse change observed during the eruption of Pavlof: a decrease in wave speed within the conduit, a lengthening of the conduit, or a suitable combination of both types of change. A decrease in intrinsic wave speed is difficult to accept given that lower wave speed in magma is usually linked with higher gas content, and the eruption at Pavlof gradually ended over the two weeks studied here (gas being a key driver of eruptions). Alternatively, since the explosions at Pavlof are understood to occur between the depths of water exsolution and magma fragmentation (Garces et al.), a lengthening of the conduit due to shallowing of the fragmentation depth or a deepening of the water exsolution depth (bottom right panel of Figure 5) can explain the observed time-lapse changes. However, there is yet another possibility based on the properties of guided waves: the decrease in wave speed can be explained by the existence of a dispersive wave known to propagate in fluid-filled cracks and conduits called a "crack wave" or a "slow wave" (Chouet). The crack wave has the property of inverse dispersion, meaning that 


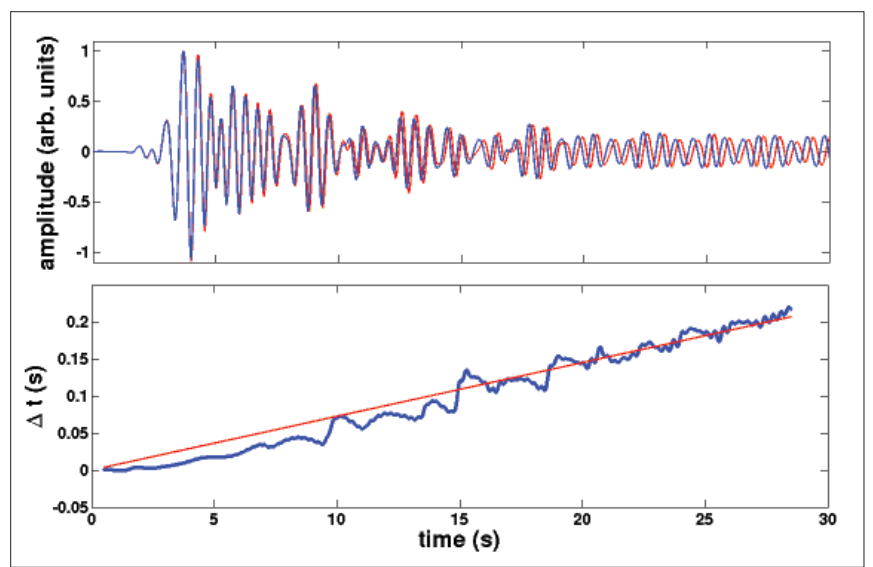

Figure 8. (top) Simulated radial particle velocity at station PVG for two conduit models, one with an acoustic wave speed of $500 \mathrm{~m} / \mathrm{s}$ (blue) and another with a wave speed 1\% slower (red). (bottom) The traveltime change, measured by CWI. The measured change is blue, and the linear fit is red. The slope of the line gives an estimated velocity change of $-0.6 \%$, somewhat lower than the true value, $-1.0 \%$.

its phase and group velocities decrease with increasing ratio of wavelength-to-conduit thickness. Therefore, a slight collapse of the conduit walls resulting from a decrease in pressure as the eruption ended offers another explanation of the observed increasing relative traveltime change in terms of a lower apparent wavespeed (bottom left panel of Figure 5). Note that this lower wave speed is not an intrinsic property of the magma, but a result of the changing geometry of the conduit waveguide.

\section{Conclusions}

In summary, we have applied CWI to repeating explosions at Pavlof volcano and conclude that the measured changes reflect subtle variations within the magma conduit. This conclusion is supported by $3 \mathrm{D}$ seismic modeling of a changing volcanic conduit at Pavlof that incorporated rugged topography and high-contrast interfaces (e.g., the conduit-rock interface). Furthermore, the modeling suggests the change calculated by CWI is an underestimate of the actual change in the geometry or velocity of the conduit. Future applications of CWI in volcanic environments must independently assess whether source or path effects dominate at a particular volcano, since the two possibilities lead to vastly different interpretations. For instance, a small change due to path effects could be indicative of increasing or decreasing stress over the entire volcanic edifice. In contrast, a small change due to a source effect is the result of dynamic processes confined to the volcanic conduit. The ability to use repeating explosions to measure the changing properties of conduits during an eruption should find applicability in the field of volcano monitoring and mitigation of hazards.

Suggested reading. "Numerical Dispersion for the Conventional Staggered-Grid Finite-Difference Elastic Wave Propagation Algorithm" by Aldridge and Haney (Sandia Technical Report SAND 2008-4991, 2008). "Elastic wave radiation from a resonating line source" by Aldridge et al. (Seismological
Society of America 2008 Annual Meeting Abstracts). "Accuracy of heterogeneous staggered-grid finite-difference modeling of Rayleigh waves" by Bohlen and Saenger (Geophysics, 2006). "Long-period volcano seismicity: Its source and use in eruption forecasting" by Chouet (Nature, 1996). "Application of wave-theoretical seismoacoustic models to the interpretation of explosion and eruption tremor signals radiated by Pavlof volcano" by Garces et al. (Journal of Geophysical Research, 2000). "Simulating seismic wave propagation in 3D elastic media using staggered-grid finite differences" by Graves (Bulletin of the Seismological Society of America, 1996). "Generalization of von Neumann analysis for a model of two discrete halfspaces: The acoustic case" by Haney (GeOpHYsics, 2007). "Numerical modeling of time-lapse monitoring of $\mathrm{CO}_{2}$ sequestration in a layered basalt reservoir" by Khatiwada et al. (SEG 2008 Expanded Abstracts). "Quantitative estimation of compaction and velocity changes using $4 \mathrm{D}$ impedance and traveltime changes" by Landro and Stammeijer (Geophysics, 2004). "Observations and analysis of B-type earthquakes, explosions, and volcanic tremor at Pavlof volcano, Alaska" by McNutt (Bulletin of the Seismological Society of America, 1986). "Coda wave interferometric detection of seismic velocity changes associated with the $1999 \mathrm{M}=3.6$ event at Mt. Vesuvius" by Pandolfi et al. (Geophysical Research Letters, 2006). "Sustained long-period seismicity at Shishaldin Volcano, Alaska" by Petersen et al. (Journal of Volcanology and Geothermal Research, 2006). "Monitoring velocity variations in the crust using earthquake doublets" by Poupinet et al. (Journal of Geophysical Research, 1984). "Finite-difference modeling of 3D seismic wave propagation in high-contrast media" by Preston et al. (SEG 2008 Expanded Abstracts). "Development of the active doublet method for measuring small velocity changes and attenuation in solids" by Roberts et al. (Journal of the Acoustical Society of America, 1992). "Coda wave interferometry for estimating nonlinear behavior in seismic velocity" by Snieder et al. (Science, 2002). "The theory of coda wave interferometry" by Snieder (Pure and Applied Geophysics, 2006). "The effects of conduit length and acoustic velocity on conduit resonance: Implications for low-frequency events" by Sturton and Neuberg (Journal of Volcanology and Geothermal Research, 2006). "Scattering behaviour at Merapi volcano (Java) revealed from an active seismic experiment" by Wegler and Luhr (Geophysical Journal International, 2001). "Increase of shear wave velocity before the 1998 eruption of Merapi volcano (Indonesia)" by Wegler et al. (Geophysical Research Letters, 2006). TZE

Acknowledgments: This work has been supported in part by the USGS Mendenhall Postdoctoral Program. We thank the Arctic Region Supercomputing Center for access to computational facilities for the finite-difference numerical simulations. Stephanie Prejean, John Power, Roel Snieder, Steve McNutt, Dave Hill, and Bernard Chouet provided helpful feedback on this work. Sandia National Laboratories is a multiprogram science and engineering facility operated by Sandia Corporation, a Lockheed-Martin company, for the U.S. Department of Energy, National Nuclear Security Administration, under contract DE-AC04-94AL85000.

Corresponding author: mhaney@usgs.gov 